\title{
Mental Health Consequences of COVID-19 Disaster
}

Gamze Özçürümez Bilgili

Department of Psychiatry, Başkent University, Ankara, Turkey

D isasters are large-scale events that are often unexpected and cause death, trauma, and destruction of property (1). Although there is no consistent definition of disasters in the literature, researchers generally agree that disasters share three key characteristics of large-scale traumatic events. First, disasters threaten to harm or death to a large group of people, regardless of the actual extent of lives lost. Second, they affect social processes, disrupting services and social networks and cause communal loss of resources. Third, they involve secondary consequences, namely identifiable mental and physical health outcomes, among those affected. In this review, the focus will be on the potential mental health consequences of COVID-19 pandemic, which fits into the definition of a disaster as outlined above.

Disaster in the context of a pandemic is "frequently marked by uncertainty, confusion and a sense of urgency" (2). Prior to, or in the early stages of a pandemic, there is widespread uncertainty about the odds and seriousness of becoming infected, along with ambiguity, and possible misinformation, about the best methods of prevention and management (3). Uncertainty may persist well into the pandemic, especially concerning the question of whether a pandemic is truly over. Therefore, like other natural disasters (e.g. floods, earthquakes, hurricanes) pandemics go against the widely held conception of a reliable world ruled by a benevolent higher power; it gives the lie to the belief of Nature as a nurturing mother or omnipotent God as a protecting parent (4). This disillusionment is clearly linked to the first key characteristic of disasters in general and COVID-19 in particular; causing great harm and becoming a leading cause of death in a large population and leaving the rest of the World under a continuing threat.

The very fact that we, as individuals and members of small groups, are very often powerless to cope with the three main sources of suffering described by Freud in 1930, in Civilization and its Discontents-namely, the frailty of our bodies, the forces of nature, and the behavior of other human beings-has made us look for comfort and aid in the larger community and its organizations (5). There is an unfailing hope in us, which stubbornly refuses to die, in the humanness of other people and social institutions. And, whenever their response-or lack of it—belies our expectations, the result is a deep feeling of utter despondence and hopelessness (6). Hence, the second key characteristic of disasters, their causing disruption of services and social net-

Corresponding Author: Gamze Özçürümez Bilgili

E-mail:

gamzeozcurumez@hotmail.com

Received: April 29, 20209 Accepted: April 30, 2020 Published: April 30, 2020

Suggested citation: Bilgili GÖ. Mental Health Consequences of COVID-19 Disaster. Infect Dis Clin Microbiol 2020; 1: 42-45.

DOI: $10.36519 / \mathrm{idcm} .2020 .0010$ 
works and communal loss of resources, is an immensely detrimental dimension of COVID-19. The increased health and economic inequalities further mark the failure of the social system to contain, nurture, care for, and protect individuals, especially the vulnerable groups. Vulnerable groups include those with pre-existing mental or physical health issues, the poor, children, young people and elderly, front-line health-care workers, recovered individuals, socially excluded groups, including prisoners, the homeless, and refugees, and those who become mentally unwell (e.g. in response to anxiety and loneliness surrounding the pandemic).

Another important dimension regarding the social system in the time of disasters is the splits and polarizations that are becoming evident. Acknowledging the fact that pandemics include not only the spread of physical illness but also the dissemination of racist, anti-migrant, homophobic propaganda and stigmatization is essential. These serve to allow aggressive desires to be simultaneously sated and projected into the other and destroyed without guilt. This is why we are witnessing not only frustration and disillusionment due to a hoped-for but lacking state and/or organizational response, but also an increase in pro-violent discourses and acts aimed at the most vulnerable groups including LGBT individuals, forced migrants, refugees, and foreigners. The social system has been created in order to make up for the fragilities, lacks, and insufficiencies of individuals and groups, and this sets a moral duty on the authorities and on the more favored members of the community to aid and care for those who are more fragile.

Coming to the third characteristic of disasters, its mental health outcomes, although many people experience emotional distress during COVID-19, there are differences in how they react to a psychosocial stressor. At one end of the spectrum, some people frankly disregard or deny the risks, and fail to engage in recommended health behaviors such as hygiene practices, and physical distancing. At the other end of the spectrum, many people react with intense anxiety or fear. A moderate level of fear or anxiety can motivate people to cope with health threats, but severe distress can be debilitating. People who are highly anxious about being infected typically go to great lengths to protect themselves. This may involve avoidance of infection-related stimuli, including people, places, and things associated with the disease. People may refuse to go to work for fear of coming in contact with infected others. Comprehensibly, worries and uncertainties about a pandemic are common, but for some, they can cause undue distress and impairment to social and occupational functioning (7). Across society, a sense of loss can stem from losing direct social contacts, and also range from loss of loved ones to loss of employment, educational opportunities, recreation, freedoms, and supports. Henceforth, some measures taken to control the pandemic might have a disproportionate effect on those most vulnerable.

A major adverse consequence of the COVID-19 pandemic is increased social isolation and loneliness which are strongly associated with anxiety, depression, self-harm, and suicide attempts across the lifespan (7). Many of the anticipated consequences of quarantine and associated social and physical distancing measures are themselves key risk factors for mental health issues. These also include alcohol and substance abuse, gambling, gender-based violence, child abuse, and psychosocial risks such as social disconnection, lack of meaning or anomie, entrapment, feeling a burden, financial stress, bereavement, loss, unemployment, homelessness, and relationship breakdown.

Repeated media consumption about COVID-19 also has certain effects on mental health. People seek trusted information via the media, which can provide swift, critical guidance regarding the pandemic. Media consumption can be adaptive and positive for mental health. However, reports of COVID-19 often use risk-elevating messages, which can amplify public anxiety. Social media can be a source of rapidly disseminated misinformation, raising perceptions of risk. Repeated media exposure to information about COVID-19 particularly can exacerbate stress responses, incite anxiety and impair functioning. Anxiety and uncertainty can drive additional media consumption and further distress, creating a vicious cycle. Media-fuelled distress can promote behaviours that negatively affect the health-care system (e.g. visits to emergency de- 
partments and hoarding of face masks), with downstream mental and physical health consequences.

Another important mental consequence of COVID-19 pandemic is mourning. Death tolls from COVID-19 are being charted, tracked, and graphed every day, but as mental health workers, one of our main concern is about the "tsunami of grief" that is just beginning as a result of these deaths. As we are all fixated on what this pandemic will mean in terms of the total lives lost, it is important to keep in mind that this number will feel far more pervasive because each life will leave multiple grieving. While grief is a very personal and unique phenomenon, it takes a well-documented physical and mental toll that can be extreme. People currently are not able to hold or attend a memorial service or funeral for their loved one. Those rituals are part of what helps us process our loss and continue on, and the majority is unable to engage in them right now. Grief is always isolating, but with people being forced to stay at home during COVID-19 pandemic, they are now getting hit with a double dose of isolation when they lose a loved one. Therefore, it is crucial to find ways to express grief and connect with others who share it. Anyone who has had to delay or postpone rituals of bereavement has to find new ways, maybe digital platforms to come together in the short term to work through their loss. But what if you are a forced migrant who has no internet access and has already experienced sadness due to separation from loved ones, grief associated with the death or disappearance of family members, and distress regarding the lack of opportunity to engage in culturally important rituals of bereavement because the place where you once called home is no more accessible or even totally demolished?

COVID-19 also intersects with rising mental health issues in childhood and adolescence. Ascertaining and mitigating the effects of school closures for youth seeking care is urgent and essential, given that school is often the first place children and adolescents seek help,as is considering vulnerabilities, such as special educational needs and developmental disorders. For the older population, promoting good mental health is important during self-isolation, which can be compounded by lifestyle restrictions, exacerbated loneliness, comorbidities (such as dementia), and feelings of worry and guilt for using resources.

First responders and health workers who come in close contact with the virus and are exposed to traumatic events, such as death and dying, while making highly challenging decisions, are also particularly at risk of stress responses. Depression, anxiety, and insomnia appear to be common consequences of the high-stress environment faced by healthcare workers amid the COVID-19 pandemic. The findings, published in JAMA Network Open as part of a study of Chinese healthcare workers, may foreshadow the mental health burden placed upon physicians, nurses, and other professionals as COVID-19 strains the medical system (8). Acknowledging this urgent need, Disasters and Mental Trauma Working Unit of Psychiatric Association of Turkey took immediate action in launching a 24/7 free mental health hotline through which volunteer psychiatrists would be available to provide frontline health workers (physicians, nurses, first responders, hospital staff, dentists, pharmacists) with some emotional personal protective equipment.

Besides the effects of COVID-19 on the general population and vulnerable groups, there are people who are actually infected by it. The patients placed in isolation are particularly prone to neuropsychiatric complications, for a number of reasons. For such individuals, isolation is evident and physical - they are confined to limited space, their movement is limited, there are contact precautions, and everyone is rushing to complete their task at hand and get out of the isolation room. Patients in isolation tend to receive less face-to-face time because a portion of patient-allocated time is spent donning and shedding the protective gear. Their isolation is deepened by the illness itself and complications arising from the infection. Those complications can include delirium, anxiety, depression, a sense of hopelessness and despair, psychological trauma (acute stress disorder or posttraumatic stress disorder [PTSD]), and cognitive impairment (9). Those affected by quarantine, regardless of their health status, are likely to report distress due to fear and risk perceptions. Besides, neuropsychiatric sequelae of surviving COVID-19, its complications, and complications associated with treatment may war- 
rant sustained mental health focus and attention. This set of sequelae may require an expansion in resources and expertise from more trauma-focused to include neuropsychiatric aspects of care in order to prevent and minimize long-term disabilities.

To optimise the effectiveness of psychiatric treatments during and after COVID-19 pandemic, targeting the factors which are both causally associated with poor mental health and modifiable by an intervention is essential (7). The optimal structure of a mentally healthy life for individuals in the wake of COVID-19 needs to be mapped out. The structure will vary as a function of background and individual circumstances. Understanding the effective, individualised ways of coping in such a situation is of paramount importance. Digital interventions for anxiety, depression, self-harm, and suicide include information provision, connectivity and triage, automated and blended therapeutic interventions (such as apps and online programmes), telephone calls and messages to reach those with poorer digital resources (digital poverty), suicide risk assess- ments, chatlines and forums, and technologies that can be used to monitor risk either passively or actively. Risks and buffers for loneliness should be a focal target in interventions to protect wellbeing. The longer-term consequences of COVID-19 for the younger and older generations (and other groups at high risk, including workers, those with existing mental health conditions, and caregivers) are also unknown and must be a priority.

In conclusion, managing concerns, fears, and misconceptions at the local community and broader public level, psychosocially supporting fellow health-care workers, optimising the effectiveness of psychiatric interventions become for us as important as treating individual patients. As psychiatrists, we have found ourselves participating in public mental health activities, helping to formulate responses to alleviate public anxiety and concerns in addition to our long fight against discrimination, stigmatization, and violence. Today, the Psychiatric Association of Turkey has a new motto: "Spread solidarity, not anxiety".
Peer-review: Externally peer-reviewed

Conflict of Interest: The authors have no conflict of interest to declare.
Financial Disclosure: The authors declared that this study has received no financial support.

\section{REFERENCES}

1 Goldmann E, Galea S. Mental health consequences of disasters Annu Rev Public Health 2014; 35: 169-83.

2 Taylor S. The psychology of pandemics: preparing for the next global outbreak of infectious disease. New Castle: Cambridge Scholars Publishing; 2019.p.5.

3 Kanadiya MK, Sallar AM. Preventive behaviors, beliefs, and anxieties in relation to the swine flu outbreak among college students aged 18-24 years. Z Gesundh Wiss 2011; 19: 139-145.

4 Khan S, Huremović D. Psychology of the pandemic. Huremović D, editor. Psychiatry of pandemics: a mental health response to infection outbreak. Switzerland: Springer; 2019.p.37-44.

5 Freud S. Civilization and its discontents. London: Hogarth Press; 1961.p.64-145.
6 Hernández TR. Social trauma: the pathogenic effects of untoward social conditions. Inter Forum Psychoanal 2006; 15: 151-156.

7 Holmes EA, O'Connor RC, Perry VH, Tracey I, Wessely S, Arseneault L, et al. Multidisciplinary research priorities for the COVID-19 pandemic: a call for action for mental health science. Lancet Psychiatry 2020; S2215-0366(20)30168-1.

8 ai J, Ma S, Wang Y, et al. Factors associated with mental health outcomes among health care workers exposed to coronavirus disease 2019. JAMA Netw Open. 2020;3:e203976.

9 Huremović D, editor. Mental health of quarantine and isolation. Psychiatry of pandemics: a mental health response to infection outbreak. Switzerland: Springer; 2019.p.95-8. 